\title{
ELECTRONIC NAVIGATIONAL CHART AS AN EQUIVALENT TO IMAGE PRODUCED BY HYPERCATADIOPTRIC CAMERA SYSTEM
}

\author{
Krzysztof Naus, Ph. D. \\ Polish Naval Academy, Poland
}

\begin{abstract}
This paper presents a dynamic hyperboloidal mapping model aimed at building image of electronic navigational chart which constitutes an equivalent to that obtained from a hypercatadioptric camera system. In the 1st part, space and three reference frames located in it are defined. These are: the observer frame and horizontal topocentric frame considered secondary (both connected with water-surface platform), and the geocentric frame, primary one. The 2nd part provides description of a way of interconnection between the observer frame and horizontal topocentric one as well as of determination of their location in relation to the geocentric reference frame, depending on course and position of water-surface platform. In the final part is presented a model of panoramic image mapping in the observer reference frame and principles of generating ENC image by using dynamic hyperboloidal mapping. Finally, conclusions indicating possible applications of the developed model are presented.
\end{abstract}

Keywords: electronic navigational chart, dynamic hyperboloidal mapping, comparative navigation

\section{INTRODUCTION}

Electronic navigational chart (ENC) is today a basic source of information on marine environment for navigator on almost every ship. On its basis he plans and monitors ship voyage. Additionally, already during the voyage, he makes use of it for conducting terrestrial navigation called also comparative observational one. He then compares selected spatial objects contained in the ENC (which represent e.g. navigational marks, coastal line edges and wave breakers, or emerging separate navigational obstacles) with those actually observed, recognizing this way a coast and next determines his ship position against the identified objects. It could be metaphorically said that performing the comparative optical navigation process he serves as a measuring instrument (e.g. an image recording camera) as well as a computer processing the recorded actual image together with selected patterns comprised in the ENC in order to compare them to each other and then to determine against the identified positions.

In the last years image recognition and its use as a basic source of information on environment has been a subject of extensive research especially in the area of photogrammetry and robotics - an interdisciplinary scientific field of mechanics, automation, electronics, cybernetics and informatics.

However maritime navigation purposes in the area of image computerized processing are satisfied only partially and deal a.o. with ENC visualization in ECDIS, comparative navigation carried out on the basis of radar image, methods of presentation of sea bed sonar images as well as evaluation of limitations in satellite positioning $[8,11,12,14]$.

Rather scarce investigations concerning image processing are focussed only on automation of the process of keeping the ship along the water path axis, on line towards a navigation mark, in a given navigation light sector, assessment of accuracy in determining navigational parameters by using CCD camera as well as optical methods for anti-terroristic protection of ships $[2,3,4,5,6,7,9,10,13]$.

It may be observed that there is a lack of scientific research in which an attempt would be made toward implementing the existing solutions or developing novel ones in the domain of computerized image processing or analytical photogrammetry, aimed at developing a comparative optical system intended for e.g. the position determining of a water-surface platform manoeuvring in coastal zone, or a panoramic optical system serving a role of the so called passive navigational radar.

This paper presents a model of ENC dynamic hyberboloidal mapping for creating an image which would be equivalent to that achievable from a hypercatadioptric camera system composed of a hyperbolic mirror and a panoramic camera CCD.

The developed ENC mapping model is based on two projection surfaces (hyperboloid and plane) and two projection centres (hyperboloid focus and camera lens focus) connected with water-surface platform.

As assumed, the model should be able first of all to serve as a theoretical basis for the dynamic hyperboloidal cartographic 
mapping of ENC, which is dedicated to comparative optical systems to be used in the future on water surface platforms. Secondly, it should provide a theoretical basis for projection of an image available from a panoramic optical system for ship traffic monitoring (which determines position and motion parameters of ships).

\section{DETERMINATION OF SPACE AND REFERENCE FRAMES}

Let $\mathrm{E}^{3}$ be 3 - dimensional Euclidian space over a body of real numbers $\mathrm{R}, E^{3}$ - associated Euclidian space, $\mathbf{O}$ - a point of $E^{3}$, a $\left(\mathbf{e}_{1}, \mathbf{e}_{2}, \mathbf{e}_{\mathbf{3}}\right)$ - an orthogonal normal base of $E^{3}$. The below written system will be called the orthogonal Cartesian reference frame (a global datum point) of $\AA^{3}$ space:

$$
\mathfrak{I}=\left\{\mathbf{O},\left(\mathbf{e}_{\mathbf{1}}, \mathbf{e}_{\mathbf{2}}, \mathbf{e}_{\mathbf{3}}\right)\right\}
$$

The point $\mathbf{O}$ will determine its origin ( or base point) and $\left(\mathbf{e}_{1}, \mathbf{e}_{2}, \mathbf{e}_{3}\right)$ - base of the system $\mathfrak{I}$.

Let $\mathfrak{I}$ be the fixed reference frame of the space $\mathrm{E}^{3}$. The set of numbers $(x, y, z)$ determines orthogonal Cartesian coordinates of the point $\mathbf{P}$ in relation to the reference frame $\mathfrak{I}$, and:

$$
\mathbf{P}=\overrightarrow{\mathbf{O P}}=x \mathbf{e}_{1}+y \mathbf{e}_{2}+z \mathbf{e}_{3} \text {, }
$$

location vector of $\mathbf{P}$ against $\mathfrak{I}$.

$$
\text { Let } \mathfrak{I}=\left\{\mathbf{O},\left(\mathbf{e}_{\mathbf{1}}, \mathbf{e}_{\mathbf{2}}, \mathbf{e}_{\mathbf{3}}\right)\right\} \text { and } \mathfrak{I}^{\prime}=\left\{\mathbf{O}^{\prime},\left(\mathbf{e}_{\mathbf{1}}^{\prime}, \mathbf{e}_{\mathbf{2}}^{\prime}, \mathbf{e}_{\mathbf{3}}^{\prime}\right)\right\}
$$
be two orthogonal Cartesian reference frames of $\mathrm{E}^{3}$ space.

The reference frame $\mathfrak{I}$ (further called geocentric one) will be connected with the Earth in such a way as its versors $\mathbf{e}_{\mathbf{1}}, \mathbf{e}_{\mathbf{2}}, \mathbf{e}_{\mathbf{3}}$ will respectively determine the axes $\mathbf{O X}, \mathbf{O Y}, \mathbf{O Z}$ of the frame. The axis will coincide with the Earth rotation axis. The remaining axes ( $\mathbf{O X}$ and $\mathbf{O Y}$ ) will lay on the Equator plane, whereas the axis $\mathbf{O X}$ will lay in the $0^{\circ}$ meridian plane, and the axis $\mathrm{OY}$ - in the $90^{\circ} \mathrm{E}$ meridian plane (also known as the Earth-centred, Earth-fixed coordinate system) [1].

The reference frame $\mathfrak{I}^{\prime}$ (further called the observer reference frame) will be connected with the water-surface platform position $\mathbf{P}$ (more precisely - with position of a panoramic optical system fixed with hull of the water-surface platform ) and its origin and base will be determined in relation to the reference frame $\mathfrak{I}$.

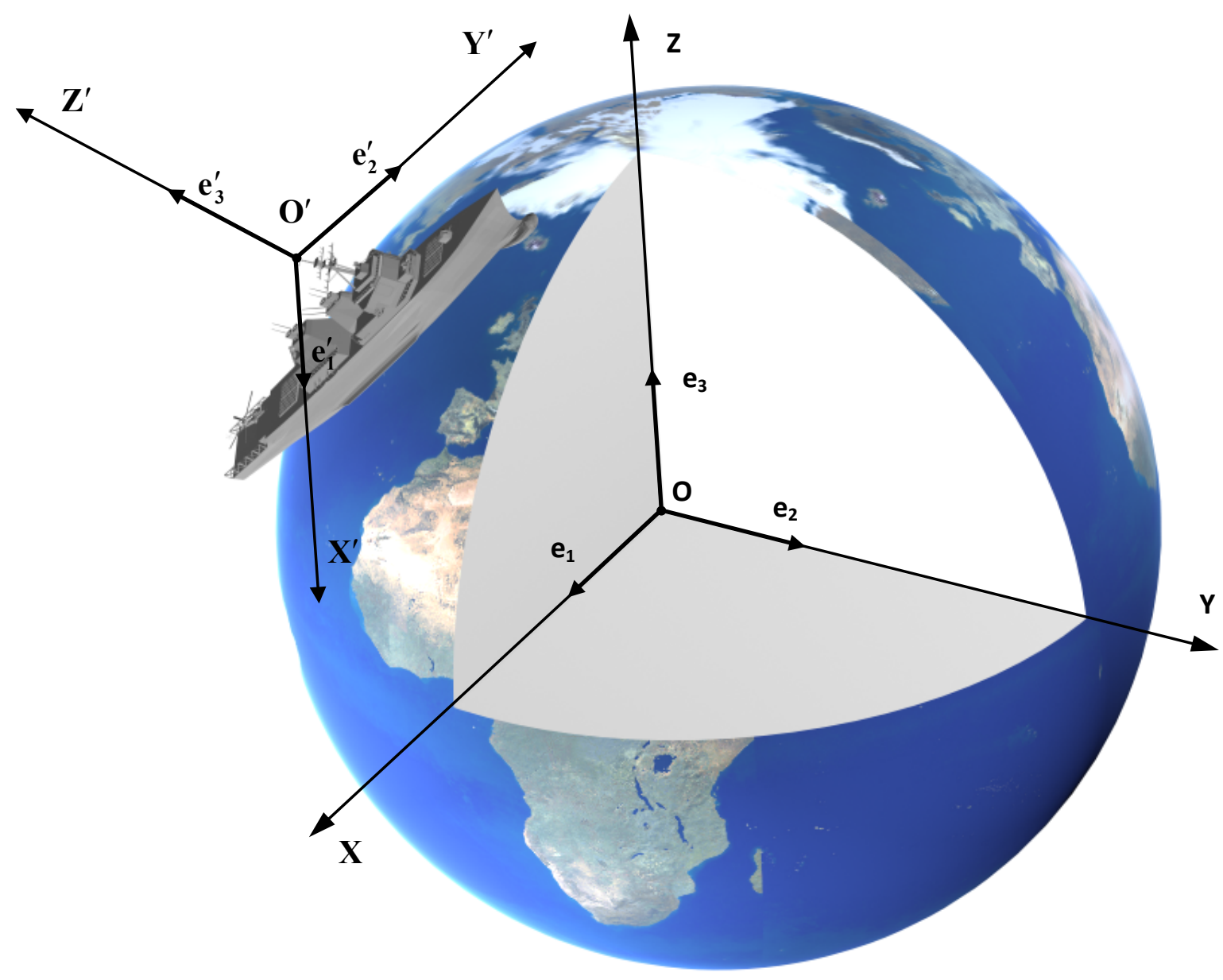

Fig. 1. Mutual arrangement of the reference frames $\mathfrak{I}^{\prime}$ and $\mathfrak{I}$ of the space $\mathrm{E}^{3}$ 
Let the observer reference frame $\mathfrak{I}^{\prime}$ be in turn connected with the horizontal topocentric reference frame $\mathfrak{I}^{\mathrm{H}}=\left\{\mathbf{O}^{\mathbf{H}},\left(\mathbf{e}_{\mathbf{1}}^{\mathbf{H}}, \mathbf{e}_{\mathbf{2}}^{\mathbf{H}}, \mathbf{e}_{\mathbf{3}}^{\mathbf{H}}\right)\right\}$ (also known as the local EastNorth-Up Cartesian coordinate system).

The horizontal topocentric reference frame $\mathfrak{J}^{\mathrm{H}}$ will be obtained as a result of transformation of $\mathfrak{I}=\left\{\mathbf{O},\left(\mathbf{e}_{1}, \mathbf{e}_{2}, \mathbf{e}_{3}\right)\right\}$. The transformation will be composed of the translation of $\left\{\mathbf{O}^{\mathbf{H}},\left(\mathbf{e}_{\mathbf{1}}^{\mathbf{H}}, \mathbf{e}_{\mathbf{2}}^{\mathbf{H}}, \mathbf{e}_{\mathbf{3}}^{\mathbf{H}}\right)\right\}$ against $\mathfrak{I}$ and the rotation of $\mathfrak{J}^{\mathbf{H}}$ with the origin $\mathbf{O}^{\mathbf{H}}=\mathbf{P}$ against the frame $\left\{\mathbf{O}^{\mathbf{H}},\left(\mathbf{e}_{\mathbf{1}}, \mathbf{e}_{\mathbf{2}}, \mathbf{e}_{\mathbf{3}}\right)\right\}$. The transformation will be described by the translation vector $\xi=\overrightarrow{\mathbf{O O}^{\mathbf{H}}}$ of the point $\mathbf{O}$ to the point $\mathbf{O}^{\mathbf{H}}$ as well as the rotation matrix $\mathbf{M}_{\mathbf{R}}$ of the base $\left(\mathbf{e}_{\mathbf{1}}^{\mathbf{H}}, \mathbf{e}_{\mathbf{2}}^{\mathbf{H}}, \mathbf{e}_{\mathbf{3}}^{\mathbf{H}}\right)$ against the base $\left(\mathbf{e}_{1}, \mathbf{e}_{\mathbf{2}}, \mathbf{e}_{3}\right)$.

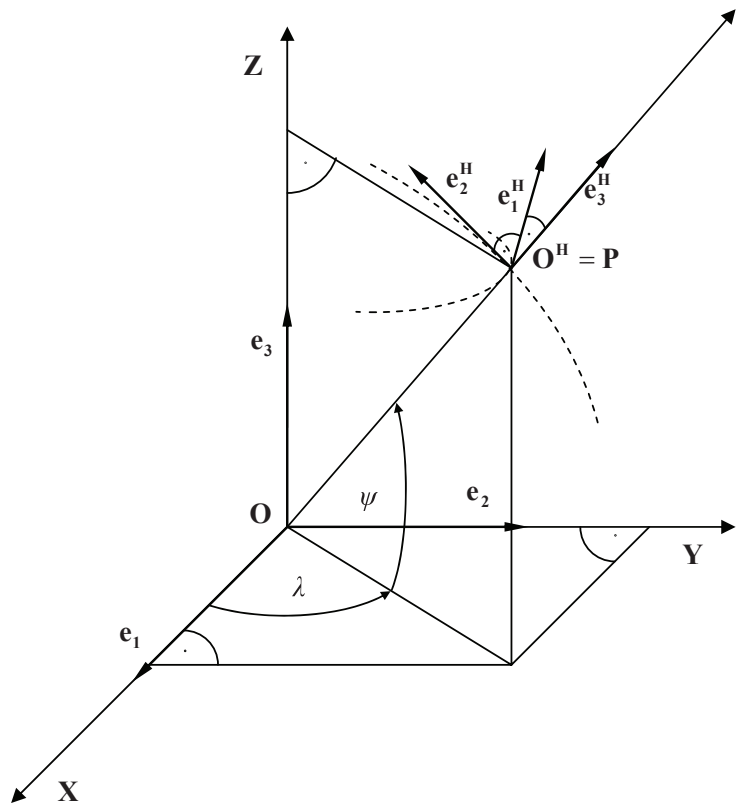

Fig. 2. Mutual arrangement of the reference frames $\mathfrak{J}_{\text {and }} \mathfrak{J}^{H}$

The base $\left(\mathbf{e}_{\mathbf{1}}^{\mathbf{H}}, \mathbf{e}_{\mathbf{2}}^{\mathbf{H}}, \mathbf{e}_{\mathbf{3}}^{\mathbf{H}}\right)$ will be formed as a result of the parametrizing of the 3-dimensional domain $\mathrm{V}$ by means of the spherical coordinates and $r, \lambda$ and $\psi$. The parametrizing function (map) of the domain $\mathrm{V}$ in relation to the reference frame $\mathfrak{I}$ will be described as follows:

$$
\mathbf{P}=r \cos \psi \cos \lambda \mathbf{e}_{1}+r \cos \psi \sin \lambda \mathbf{e}_{2}+r \sin \psi \mathbf{e}_{\mathbf{3}} .
$$

As a result of calculation of derivatives of the function (3) against $\lambda, \psi, r$, the following local base vectors in the three-dimensional domain $\mathrm{V}$ will be achieved:

$$
\begin{gathered}
\mathbf{g}_{\lambda}=-r \cos \psi \sin \lambda \mathbf{e}_{1}+r \cos \psi \cos \lambda \mathbf{e}_{2}, \\
\mathbf{g}_{\psi}=-r \cos \psi \cos \lambda \mathbf{e}_{1}-r \sin \psi \sin \lambda \mathbf{e}_{2}+r \cos \psi \mathbf{e}_{3}, \\
\mathbf{g}_{r}=\cos \psi \cos \lambda \mathbf{e}_{1}+\cos \psi \sin \lambda \mathbf{e}_{2}+\sin \psi \mathbf{e}_{3},
\end{gathered}
$$

which, after their normalization

$$
\mathbf{e}_{1}^{\mathbf{H}}=\frac{\mathbf{g}_{\lambda}}{\left|\mathbf{g}_{\lambda}\right|}=-\sin \lambda \mathbf{e}_{1}+\cos \lambda \mathbf{e}_{2},
$$

$\mathbf{e}_{2}^{\mathbf{H}}=\frac{\mathbf{g}_{\psi}}{\left|\mathbf{g}_{\psi}\right|}=-\sin \psi \cos \lambda \mathbf{e}_{1}-\sin \psi \sin \lambda \mathbf{e}_{2}+\cos \psi \mathbf{e}_{3}$,

$\mathbf{e}_{3}^{\mathbf{H}}=\frac{\mathbf{g}_{r}}{\left|\mathbf{g}_{r}\right|}=\cos \psi \cos \lambda \mathbf{e}_{1}+\cos \psi \sin \lambda \mathbf{e}_{2}+\sin \psi \mathbf{e}_{3}$,

will constitute the base $\mathfrak{I}^{\mathrm{H}}$, for every point on the Earth surface, of known geocentric latitude $\psi$ and geographic longitude $\lambda$. In order to facilitate further calculations the rotation of the base of the reference frame $\mathfrak{I}$ against the base $\mathfrak{I}^{\mathrm{H}}$ will be described by means of the complex matrix of rotation as follows:

$\mathbf{M}_{\mathbf{R}}^{(\lambda, \psi)}=\left[\begin{array}{cccc}-\sin \lambda & \cos \lambda & 0 & 0 \\ -\sin \psi \cos \lambda & -\sin \psi \sin \lambda & \cos \psi & 0 \\ \cos \psi \cos \lambda & \cos \psi \sin \lambda & \sin \psi & 0 \\ 0 & 0 & 0 & 1\end{array}\right]$

where:

$\psi$ - geocentric latitude of the point $\mathbf{P}$,

$\lambda$ - geographic longitude of the point $\mathbf{P}$.

\section{CONNECTION OF THE HORIZONTAL TOPOCENTRIC REFERENCE FRAME WITH WATER-SURFACE PLATFORM}

Let be known an actual heading $H D G$ and position (the geocentric latitude $\psi$ geographic longitude $\lambda$ ) of the point $\mathbf{P}$ in relation to the reference frame $\mathfrak{I}$. Let the point $\mathbf{P}$ determine the centres of $\mathfrak{I}^{\mathrm{H}}$ and $\mathfrak{I}^{\prime}$, and $A_{O}=H D G$ change sense of the versors $\mathbf{e}_{1}^{\mathbf{H}}$ and $\mathbf{e}_{2}^{\mathbf{H}}$ (at unchangeable $\mathbf{e}_{3}^{\mathbf{H}}$ ) of the base of horizontal topocentric reference frame $\mathfrak{I}^{\mathbf{H}}$ by means of the following matrix of rotation:

$$
\mathbf{M}_{\mathbf{R}}^{\left(A_{O}\right)}=\left[\begin{array}{cccc}
\cos A_{O} & \sin A_{O} & 0 & 0 \\
-\sin A_{O} & \cos A_{O} & 0 & 0 \\
0 & 0 & 1 & 0 \\
0 & 0 & 0 & 1
\end{array}\right],
$$

determining this way the base $\left(\mathbf{e}_{1}^{\prime}, \mathbf{e}_{2}^{\prime}, \mathbf{e}_{\mathbf{3}}^{\prime}\right)$ of the observer reference frame $\mathfrak{I}^{\prime}$. 


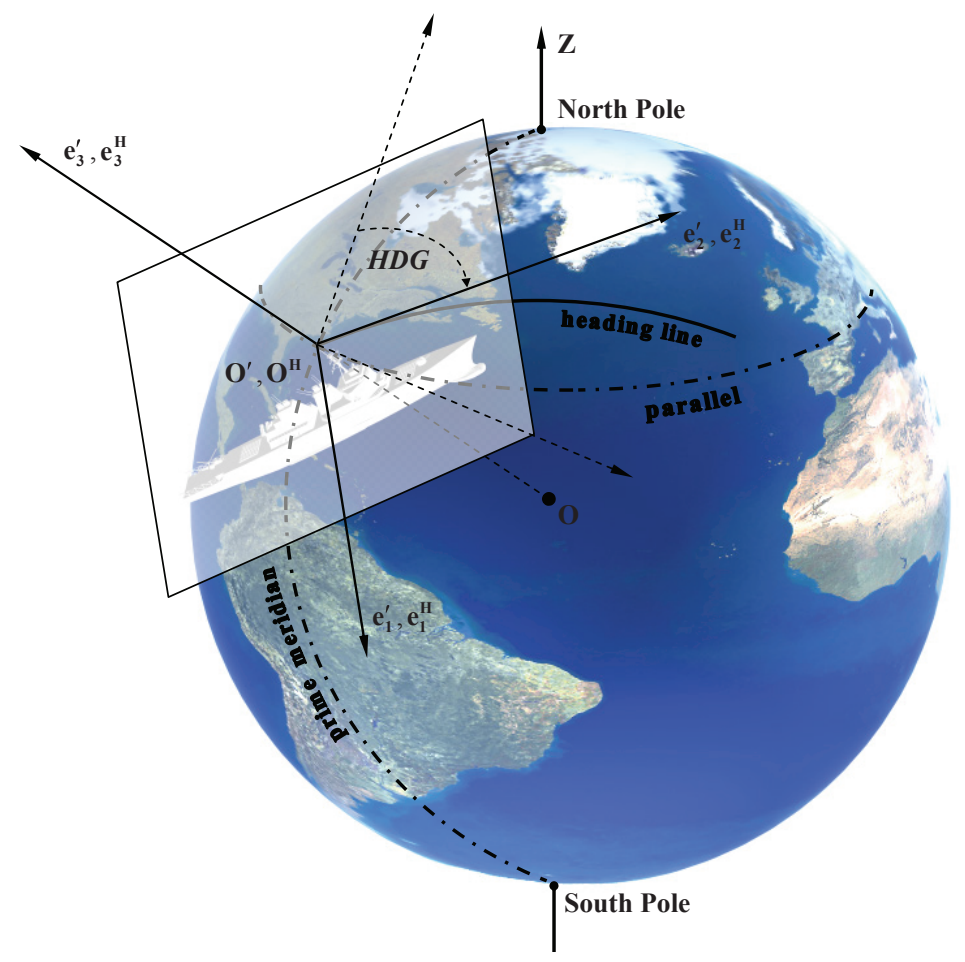

Fig. 3. Graphical interpretation of transformation of the base of the horizontal topocentric reference frame $\mathfrak{I}^{\mathrm{H}}$ into the base of the observer reference frame $\mathfrak{I}^{\prime}$

The transformation of the base of the reference frame $\mathfrak{J}$, successively into the base of $\mathfrak{I}^{\mathrm{H}}, \mathfrak{I}^{\prime}$, will be described by means of the following complex matrix of rotation:

$\mathbf{M}_{\mathbf{R}}^{\left(\lambda, \psi, A_{O}\right)}=\mathbf{M}_{\mathbf{R}}^{\left(A_{O}\right)} \mathbf{M}_{\mathbf{R}}^{(\lambda, \psi)}=\left[\begin{array}{cccc}-\sin A_{O} \sin \psi \cos \lambda-\cos A_{O} \sin \lambda & \cos A_{O} \cos \lambda-\sin A_{O} \sin \psi \sin \lambda & \sin A_{O} \cos \psi & 0 \\ -\sin A_{O} \sin \lambda-\cos A_{O} \sin \lambda \cos \lambda & -\sin A_{O} \cos \lambda-\cos A_{O} \sin \psi \sin \lambda & \cos A_{O} \cos \psi & 0 \\ \cos \psi \cos \lambda & \cos \psi \sin \lambda & \sin \psi & 0 \\ 0 & 0 & 0 & 1\end{array}\right]$.

As assumed, all geometrical transformations will be conducted on the basis of homogeneous, orthogonal Cartesian coordinates which enable to join rotations and simultaneously to scale (dilatation) with translation. In the case of the examined transformations, the assumption will make it possible to join together the rotation matrix $\mathbf{M}_{\mathbf{R}}^{\left(\lambda, \psi, A_{O}\right)}$ and translation matrix $\mathbf{M}_{\mathbf{T}}^{(\zeta)}$ ( which represents the vector $\xi=\overrightarrow{\mathbf{O O}^{\mathbf{H}}}$ ). As a result of combining the two transformations the final complex matrix of geometrical transformations, $\mathbf{M}_{\Im \rightarrow \Im^{\prime}}$, which enables to transform the base and origin of $\mathfrak{I}=\left\{\mathbf{O},\left(\mathbf{e}_{\mathbf{1}}, \mathbf{e}_{\mathbf{2}}, \mathbf{e}_{\mathbf{3}}\right)\right\}$ into the base and origin of $\mathfrak{I}^{\prime}=\left\{\mathbf{O}^{\prime},\left(\mathbf{e}_{\mathbf{1}}^{\prime}, \mathbf{e}_{\mathbf{2}}^{\prime}, \mathbf{e}_{\mathbf{3}}^{\prime}\right)\right\}$, will be obtained:

$$
\begin{aligned}
& \mathbf{M}_{\mathfrak{I} \rightarrow \mathfrak{I}^{\prime}}=\mathbf{M}_{\mathbf{T}}^{(\zeta)} \mathbf{M}_{\mathbf{R}}^{\left(\lambda, \psi, A_{0}\right)}= \\
& =\left[\begin{array}{cccc}
x_{1}^{\prime} & y_{1}^{\prime} & z_{1}^{\prime} & -\left(x_{1}^{\prime} x_{0}+y_{1}^{\prime} y_{0}+z_{1}^{\prime} z_{0}\right) \\
x_{2}^{\prime} & y_{2}^{\prime} & z_{2}^{\prime} & -\left(x_{2}^{\prime} x_{0}+y_{2}^{\prime} y_{0}+z_{2}^{\prime} z_{0}\right) \\
x_{3}^{\prime} & y_{3}^{\prime} & z_{3}^{\prime} & -\left(x_{3}^{\prime} x_{0}+y_{3}^{\prime} y_{0}+z_{3}^{\prime} z_{0}\right) \\
0 & 0 & 0 & 1
\end{array}\right],
\end{aligned}
$$

where:

$$
\begin{aligned}
\mathbf{M}_{\mathbf{T}}^{(\zeta)}= & {\left[\begin{array}{cccc}
1 & 0 & 0 & -x_{0} \\
0 & 1 & 0 & -y_{0} \\
0 & 0 & 1 & -z_{0} \\
0 & 0 & 0 & 1
\end{array}\right] \text { point } \mathbf{O} \text { into the point } \mathbf{O}^{\prime}=\mathbf{O}^{\mathbf{H}}=\mathbf{P} } \\
\mathbf{e}_{1}^{\prime}= & \left(x_{1}^{\prime}, y_{1}^{\prime}, z_{1}^{\prime}\right), \\
& x_{1}^{\prime}=-\sin A_{O} \sin \psi \cos \lambda-\cos A_{O} \sin \lambda \\
& y_{1}^{\prime}=\cos A_{O} \cos \lambda-\sin A_{O} \sin \psi \sin \lambda \\
& z_{1}^{\prime}=\sin A_{O} \cos \psi ; \\
\mathbf{e}_{2}^{\prime}= & \left(x_{2}^{\prime}, y_{2}^{\prime}, z_{2}^{\prime}\right), \\
& x_{2}^{\prime}=-\sin A_{O} \sin \lambda-\cos A_{O} \sin \lambda \cos \lambda \\
& y_{2}^{\prime}=-\sin A_{O} \cos \lambda-\cos A_{O} \sin \psi \sin \lambda \\
& z_{2}^{\prime}=\cos A_{O} \cos \psi ;
\end{aligned}
$$




$$
\begin{aligned}
\mathbf{e}_{3}^{\prime}=\left(x_{3}^{\prime}, y_{3}^{\prime}, z_{3}^{\prime}\right), & \\
x_{3}^{\prime} & =\cos \psi \cos \lambda, \\
y_{3}^{\prime} & =\cos \psi \sin \lambda, \\
z_{3}^{\prime} & =\sin \psi ; \\
\mathbf{O}^{\prime}=\mathbf{O}^{\mathbf{H}} & =\mathbf{P}=\left(x_{0}, y_{0}, z_{0}\right) .
\end{aligned}
$$

\section{MODEL OF THE PANORAMIC IMAGE MAPPING IN THE OBSERVER REFERENCE FRAME}

On the basis of the assumption that image should be generated around a ship of changeable position it is proposed to rigidly fix a hypercatadioptric camera system with the observer reference frame $\mathfrak{I}^{\prime}$.

A position of hyperboloidal mirror surface $\mathrm{S}_{\mathrm{m}}$ against $\mathfrak{I}^{\prime}$ will be determined by using the following relations:

$\mathrm{S}_{\mathrm{m}}=\left\{\begin{array}{l}\mathbf{P}^{\mathrm{m}} \in \mathrm{E}^{3}, \mathbf{P}^{\mathrm{m}}=\mathbf{O}^{\prime}+x^{\prime} \mathbf{e}_{1}^{\prime}+y^{\prime} \mathbf{e}_{2}^{\prime}+z^{\prime} \mathbf{e}_{3}^{\prime},\left(x^{\prime}, y^{\prime}, z^{\prime}\right) \in \mathrm{R}^{3} \\ \left(x^{\prime}, y^{\prime}, z^{\prime}\right) \mid \frac{\left(z^{\prime}-c_{m}\right)^{2}}{a_{m}}-\frac{x^{\prime 2}+y^{\prime 2}}{b_{m}}=1\end{array}\right\}$

where:

$c_{m}=\sqrt{a_{m}^{2}+b_{m}^{2}}$,

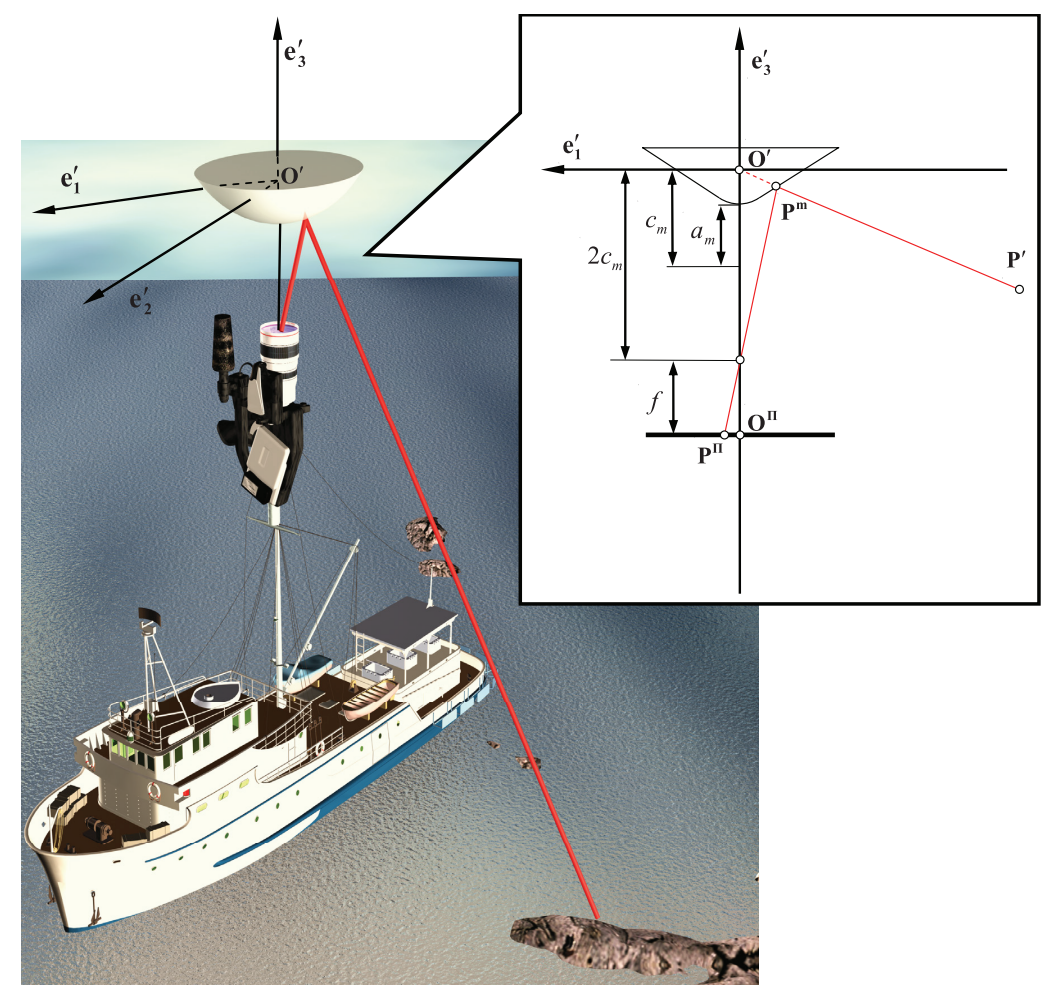

Fig. 4. Arrangement of the optical system elements in relation to the $\mathfrak{I}^{\prime}$ and the hypercatadioptric projection model $a_{m}$ - is the semi-major axis of the hyperbola,

$b_{m}$ - is the semi-minor axis of the hyperbola.

And, the relations:

$\Pi=\left\{\begin{array}{l}\mathbf{P}^{\Pi} \in \mathrm{E}^{3}, \mathbf{P}^{\Pi}=\mathbf{O}^{\prime}+x^{\prime} \mathbf{e}_{\mathbf{1}}^{\prime}+y^{\prime} \mathbf{e}_{2}^{\prime}+\left(2 c_{m}+f\right) \mathbf{e}_{3}^{\prime},\left(x^{\prime}, y^{\prime}\right) \in \mathrm{R}^{2} \\ \mathbf{O}^{\Pi}=\left(0,0,2 c_{m}+f\right) \\ \mathfrak{J}^{\Pi}=\left\{\mathbf{O}^{\Pi},\left(\mathbf{e}_{1}^{\prime}, \mathbf{e}_{2}^{\prime}\right)\right\}\end{array}\right\}$,

will determine, against the $\mathfrak{I}^{\prime}$, the projection plane $\prod$ (of CCD camera matrix), the focal point $\mathbf{O}^{\Pi}$ and the reference frame of projection plane $\mathfrak{J}^{\Pi}$, where $f$ is the focal length of camera .

The mapping of an arbitrary point $\mathbf{P}^{\prime}=\left[x^{\prime}, y^{\prime}, z^{\prime}, 1\right]^{\mathrm{T}}$ into the point $\mathbf{P}^{\mathbf{m}}=\left[x^{m}, y^{m}, z^{m}, 1\right]^{\mathrm{T}}$ located on the hyperboloidal mirror surface $\mathrm{S}_{\mathrm{m}}$ will be conducted by means of the mapping matrix I:

$$
\mathbf{M}_{\mathfrak{I}^{\prime} \rightarrow \mathbf{S}_{\mathbf{m}}}=\left[\begin{array}{cccc}
k_{m} & 0 & 0 & 0 \\
0 & k_{m} & 0 & 0 \\
0 & 0 & k_{m} & 0 \\
0 & 0 & 0 & 1
\end{array}\right]
$$

where:

$$
k_{m}=\frac{b_{m}^{2}}{a_{m}\left|\mathbf{P}^{\prime}\right|-c_{m} z^{\prime}} \mathbf{P}^{\prime} .
$$

The next, successive mapping of the point $\mathbf{P}^{\mathbf{m}}=\left[x^{m}, y^{m}, z^{m}, 1\right]^{\mathrm{T}}$ located on the hyperboloidal mirror surface $\mathrm{S}_{\mathrm{m}}$ into the point $\mathbf{P}^{\Pi}=\left[x^{\Pi}, y^{\Pi}, z^{\Pi}, 1\right]^{\mathrm{T}}$ located on the projection plane $\Pi$ will be performed by using the mapping matrix II:

$$
\mathbf{M}_{S m \rightarrow \Im^{\Pi}}=\left[\begin{array}{cccc}
k_{\Pi} & 0 & 0 & 0 \\
0 & k_{\Pi} & 0 & 0 \\
0 & 0 & k_{\Pi} & 0 \\
0 & 0 & 0 & 1
\end{array}\right],
$$

where:

$$
k_{\Pi}=\frac{f}{z^{m}+2 c_{m}} \mathbf{P}^{\mathbf{m}} .
$$


The image of the point $\Pi$ achieved on the projection plane $\Pi$ will be inverted by $180^{\circ}$ against $\mathbf{e}_{3}^{\prime}$. Owing to this its spatial orientation will coincide with the actual heading $H D G$ of the water-surface platform.

To facilitate the calculations it was assumed that the mapping $\mathbf{P}^{\prime} \rightarrow \mathbf{P}^{\mathbf{m}} \rightarrow \mathbf{P}^{\Pi}$ will be performed by means of a single mapping matrix combined of the mapping matrix I and the mapping matrix II:

$$
\mathbf{M}_{\mathfrak{\Im}^{\prime} \rightarrow \mathfrak{\Im}^{\Pi}}=\left[\begin{array}{cccc}
k_{m \Pi} & 0 & 0 & 0 \\
0 & k_{m \Pi} & 0 & 0 \\
0 & 0 & k_{m \Pi} & 0 \\
0 & 0 & 0 & 1
\end{array}\right],
$$

where:

$$
k_{m I}=\frac{-b_{m}^{2} f}{\left(a_{m}^{2}+c_{m}^{2}\right) z^{\prime}+2 a_{m} c_{m}\left|\mathbf{P}^{\prime}\right|} .
$$

\section{PRODUCING AN IMAGE OF ELECTRONIC NAVIGATIONAL CHART IN DYNAMIC HYPERBOLOIDAL MAPPING}

Producing process of ENC image on the water surface platform in motion must be preceded by determination of its actual heading $H D G$, e.g. by using a gyro-compass , as well as its position $\mathbf{P}$ (usually described by the ellipsoidal coordinates: $\varphi, \lambda$ and, additionally, by a pre-set ellipsoidal height $H$ of CCD camera matrix) against the $\mathfrak{I}$, e.g. with the use of an inertial system. On the basis of the obtained parameters, should be determined $\mathfrak{I}$ - origin coordinates against the $\mathfrak{I}$, expressed in the geocentric orthogonal Cartesian coordinates and spherical ones. To this end the following relations may be used:

$$
\begin{gathered}
\left.\mathbf{P}=\left[\begin{array}{l}
x_{0} \\
y_{0} \\
z_{0}
\end{array}\right]=\left[\begin{array}{c}
\left(\frac{a}{\sqrt{1-e^{2} \cdot \sin ^{2} \varphi}}+H\right) \cdot \cos \varphi \cdot \cos \lambda \\
\left(\frac{a}{\sqrt{1-e^{2} \cdot \sin ^{2} \varphi}}+H\right) \cdot \cos \varphi \cdot \sin \lambda \\
{\left[\frac{a}{\sqrt{1-e^{2} \cdot \sin ^{2} \varphi}} \cdot\left(1-e^{2}\right)+H\right.}
\end{array}\right] \cdot \sin \varphi\right] \\
\mathbf{P}=\left[\begin{array}{l}
\psi \\
\lambda
\end{array}\right]=\left[\begin{array}{c}
\left.\operatorname{arctg}\left(\frac{z_{0}}{\sqrt{x_{0}^{2}+y_{0}^{2}}}\right)\right], \\
\lambda
\end{array}\right]
\end{gathered}
$$

where : $a$ - length of semi-major axis of reference ellipsoid, $e^{2}$ - square of its 1 st eccentric (for WGS-84 ellipsoid $a=6378137.0 \mathrm{~m}$ and $e^{2}=0.006943800229$ should be taken). On the basis of the orthogonal Cartesian coordinates $\left(x_{0}, y_{0}, z_{0}\right)$ and the spherical ones $(\psi, \lambda)$ of the point $\mathbf{P}$, obtained this way, as well as the actual heading $A_{O}=H D G$, elements of the complex matrix $\mathbf{M}_{\mathfrak{I} \rightarrow \mathfrak{I}^{\prime}}$ transforming $\mathfrak{I}$ into $\mathfrak{I}^{\prime}$ (13) should be developed.

Next, by applying the relation (22 ), the ellipsoidal coordinates $\left(\varphi_{1}, \lambda_{1}, H_{1}=0\right)$ should be changed into the orthogonal Cartesian coordinates $\left(x_{1}, y_{1}, z_{1}\right)$ of all the points which represent ( in a vectorial form ) geometrical objects contained in ENC. The so changed coordinates describing geometrical objects in orthogonal Cartesian coordinates in relation to $\mathfrak{I}$, should be transformed into $\mathfrak{I}^{\prime}$ by using the matrix $\mathbf{M}_{\mathfrak{I} \rightarrow \mathfrak{I}^{\prime}}$.

After transformation of all coordinates of ENC geometrical objects into the reference frame $\mathfrak{I}^{\prime}$, the final, based on them, operation which results in the creating of the "map-like" image on the hyperboloidal mirror and, subsequently, on the CCD camera matrix, by means of the matrix $\mathbf{M}_{\mathfrak{\Im}^{\prime} \rightarrow \Im^{I}}$ (20), will be performed.

Fig. 5 presents two "map-like" images (including only coastal line edges) generated with the use of the application developed by this author, the first - in the Mercator mapping system and the other - in the dynamic hyperboloidal mapping system, both based on the ENC basic cell of port of Gdynia („PLGDYNA.000”).

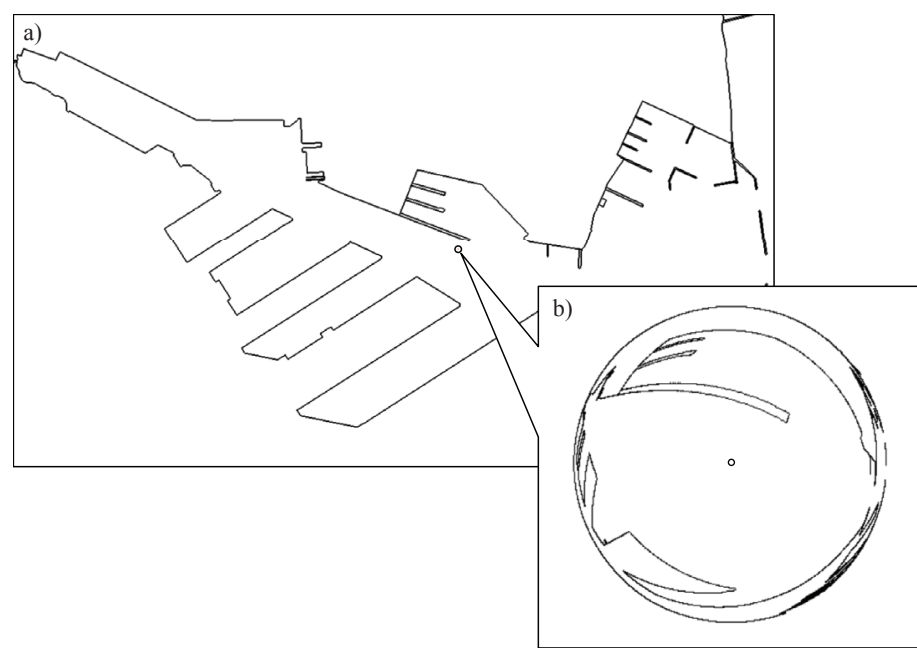

Fig. 5. ENC map-like images of port of Gdynia, generated: a) in the Mercator mapping system, $b$ ) in the dynamic hyperboloidal mapping system

\section{CONCLUSIONS}

The developed mapping model may be implemented In the future into optical comparative systems as well as panoramic optical systems for ship traffic monitoring.

The optical comparative system could be applied on water-surface platforms manoeuvring in coastal zone (including unattended platforms), as an autonomic system or that supporting other navigational systems (e.g. Loran C or GPS) in circumstances which exclude any use of the above mentioned systems. 
The panoramic optical system for ship traffic monitoring could be installed on water-surface platforms as well as land-based systems for ship traffic control and supervision (i.e. Vessel Traffic Service - VTS) in good visibility conditions, also as an autonomic system or that supporting other radar system. The developed cartographic mapping system is untypical because in it two projection surfaces (of hyperboloidal mirror and CCD matrix plane) and two projection centres (hyperboloid focus and camera lens focus) are used, as well as due to the fixing of the projection system with the water-surface platform (this is why it is named "dynamic mapping"). For this reason any attempt to apply the mapping in the optical system for ship traffic monitoring should be preceded by additional investigations connected with assessment of a character of its mapping ability distortions (especially concerning distance).

A research team of Navigation and Marine Hydrography Institute, Polish Naval Academy, presently carries out assessment tests on usability of the developed ENC mapping model for optical comparative methods of position determination of water-surface platform (also autonomic one) in harbour areas as well as usability of optical comparative methods for determination of water-surface platform position against quay during mooring (unmooring) manoeuvre.

Preliminary results of the tests in question are promising and - after completing whole the project - its results will be submitted for publication.

\section{BIBLIOGRAPHY}

1. Felski A.: A concept of navigation within orthogonal Cartesian space (in Polish), Zeszyt Naukowy Akademii Marynarki Wojennej ( Scientific bulletin of Polish Naval Academy) No. 110 A, Gdynia 1991

2. Franklin D. Snyder, Daniel D. Morris, Paul H. Haley, Robert Collins, Andrea M.: Autonomous River Navigation. Proceedings of SPIE, Mobile Robots XVII, 2004, pp. $221-232$

3. Künzner N., Kushauer J., Katzenbeiier S., Wingender K.: Modern electro-optical imaging system for maritime surveillance applications, Waterside Security Conf. (WSS) , 2010, pp. $1-4$

4. Hoshizaki T., Andrisani II D., Braun A. W., Mulyana A. K., Bethel J. S.: Performance of integrated electro-optical navigation systems. The Journal of Navigation, Vol. 51, No. 2, 2004, pp. $101-129$

5. Naus K., Wąż M.: Accuracy in fixing ship's positions by camera survey of bearings, Geodesy and Cartography, 2011, Vol. 5, No 1, pp. $61-73$

6. Naus K., Wąż M.: Measurement accuracy of distance between two CCD cameras ( in Polish), Zeszyt Naukowy Akademii Marynarki Wojennej ( Scientific bulletin of Polish Naval Academy) No. 4 (187), Gdynia 2011, pp. $73-82$
7. Naus K.: Accuracy in fixing ship's positions by CCD camera survey of horizontal angles, Geomatics and Environmental Engineering, No. 4, pp. 47 - 61

8. Naus K., Wąż M.: A Simplified navigational chart pyramid dedicated to an autonomous navigational system. Polish Hyperbaric Research, Vol. 40, No. 3, 2012, pp. 139-161, ISSN 1734-7009

9. Nowak A.: Protection Level of "Snapshot" RAIM Methods in Poor Geometry of Satellites, Advances in Computer Science - Network Centric Warfare, ISBN 978-83-922739-8-1, 2009, pp.156-162

10. Nowak A.: Influence of Pseudo-range Measurement Errors and Space Segment Geometry on GPS Fixes Distribution. Advances in Computer Science - Network Centric Warfare, ISBN 978-83-922739-8-1, 2009, pp.148 $-154$

11. Szulc D., Narloch A.: Influence of variogram on credibility of 3D model of terrain in kriging method (in Polish). Zeszyt Naukowy Akademii Marynarki Wojennej ( Scientific bulletin of Polish Naval Academy) No. 4 (183), Gdynia 2011, pp. 113 - 123

12. Szulc D.: Analysis of sonar images for ensuring safe stay of ship in port ( in Polish), Logistyka No. 6, 2009, pp. $117-123$

13. Ryynanen K., Vehkaoja A., Osterberg P., Joro R.: Automatic recognition of sector light boundaries based on digital imaging. IALA Bulletin, Issue 1/2007, pp. 30 $-33$

14. Wąż M.: Problems with Precise Matching Radar Image to the Nautical Chart, Annual of Navigation 16, 2010, pp. $149-164$

15. Wąż M.: Navigation based on characteristic points from radar image, Scientific Journals of Maritime University of Szczecin, 20 (92), 2010, pp. 140 - 145.

\section{CONTACT WITHE THE AUTHOR}

Krzysztof Naus

Polish Naval Academy 69 Śmidowicza St. 81-103 Gdynia Poland 